\title{
Single Center Review of Femoral Arteriovenous Grafts for Hemodialysis
}

\author{
Michael J. Englesbe, MD, Wajd N. Al-Holou, Alice T. Moyer, Jessica Robbins, MD, \\ Shawn J. Pelletier, MD, John Magee, MD, Randall S. Sung, MD, Darrell Campbell, MD, \\ Jeffery D. Punch, MD
}

Department of Surgery, Section of Transplantation, University of Michigan Health System, 2926 Taubman Center, 1500 E Medical Center Drive, Ann Arbor, Michigan, 48109-0331, USA

\begin{abstract}
Introduction: It is unclear how to manage high risk hemodialysis patients who present with an indwelling catheter. The National Kidney Foundation Practice Guidelines urge prompt removal of the catheter, but the guidelines do not specifically address the problem of patients whose only option is a femoral arteriovenous (AV) graft.

Methods: This study was a retrospective review of all patients who underwent femoral AV graft placement for hemodialysis access between January 1, 1996 and January 1, 2003 at the University of Michigan Health System (UMHS). Graft patency is reported according to the standards developed by the Society of Vascular Surgery and the American Association of Vascular Surgeons.

Results: Thirty patients were identified who had undergone femoral AV graft placement. The mean follow-up was 23 months (range 1-75 months). The patients had had significant medical comorbidities and multiple previous access operations (mean 3; interquartile range 1-5). The 1-year secondary graft patency rate was $41 \%$, the 2 -year rate was $26 \%$, and the 3 -year rate was $21 \%$. Infection was the cause of final graft loss in eight patients $(50 \%$ of the grafts losses, $27 \%$ of the total grafts placed.) Among those who died $(n=14)$, the mean time from femoral graft placement to death was $31.2 \pm 27.5$ months. The patient survival was quite low: at 1 year $81 \%$, at 2 years $68 \%$, and at 3 years $54 \%$.

Conclusions: These complex patients who have exhausted their upper extremity hemodialysis options do poorly following femoral AV graft placement. Consideration should be given to longterm catheter-based access in some of these patients.
\end{abstract}

$\mathrm{P}$ atients who are no longer candidates for upper extremity hemodialysis arteriovenous (AV) grafts or fistulas present a difficult problem. These patients usually have had multiple previous access surgeries, have been in renal failure for extended periods of time, and have

Correspondence to: Michael J. Englesbe, MD, Department of Surgery, Section of Transplantation, University of Michigan Health System, 2926 Taubman Center, 1500 E Medical Center Drive, Ann Arbor, Michigan, 48109-0331, USA, e-mail: englesbe@med.umich.edu multiple, severe medical co-morbidities. In our experience, these patients rarely have peritoneal dialysis or transplantation as an option. Surgeons generally agree that upper extremity hemodialysis options are preferred. There are encouraging reports of good results with femoral AV grafts for hemodialysis, ${ }^{1,2}$ as well as reports of relatively poor outcomes with these grafts. ${ }^{3,4}$

In our experience, these patients are uniquely at high risk with significant short-term mortality. Luckily, with aggressive measures to find alternatives to femoral access, such 
as angiographic recanalization of central veins, few patients require femoral $\mathrm{AV}$ grafts for hemodialysis access. It is unclear how to deal with the very high risk patient who presents with an indwelling catheter. The National Kidney Foundation Practice Guidelines suggest removing the catheter in most patients, but the guidelines do not specifically address femoral AV graft access. For the sake of improving the care of these complex patients, we report our experience with femoral AV grafts using the reporting standards of the Society of Vascular Surgeons and the American Association of Vascular Surgeons (SVS/AAVS).

\section{METHODS}

Patients in this study included adults who underwent femoral AV graft placement between January 1, 1996 and January 1, 2003 at the University of Michigan Health System (UMHS). Permission was obtained from our institutional human subjects committee. Patients were identified through review of the medical records. Data obtained included demographic information (gender and age), baseline clinical information (type of disease leading to end-stage renal failure, medical co-morbidities, number of previous access operations, the reason for femoral access), data on the access operation (date of operation, inflow vessel used, outflow vessel used, size of graft used), and information on the postoperative course (complications, date and reason for graft failure, number and efficacy of graft salvage procedures).

Baseline characteristics were compared using Fisher's exact test for dichotomous variables and the Wilcoxon rank-sum test for continuous variables. Primary and secondary patency is reported according to standards developed by the SVS/AAVS. Primary graft failure requires an intervention to reinstate patency at an access site, including surgical interventions such as thrombectomy. Final graft failure precipitates abandoning an access site. Primary patency continues until primary graft failure. Secondary patency continues until final graft failure.

Actuarial graft patency and patient survival were determined using the Kaplan-Meier method. Groups were compared by the log-rank test. Statistical analysis was performed using SAS version 8.0 (SAS Institute, Cary, NC, USA).

\section{RESULTS}

Thirty patients were identified who underwent femoral AV graft placement. The mean follow-up was 23 months
Table 1.

Baseline characteristics

\begin{tabular}{lr}
\hline \multicolumn{1}{c}{ Characteristic } & No. \\
\hline Hypertension & $17(57 \%)$ \\
Coronary artery disease & $16(53 \%)$ \\
Obesity (BMI > 40) & $15(50 \%)$ \\
Diabetes & $10(33 \%)$ \\
Deep venous thrombosis & $5(17 \%)$ \\
Hyperlipidemia & $4(13 \%)$ \\
Peripheral vascular disease & $3(10 \%)$ \\
\hline
\end{tabular}

BMI: body mass index.

(range 1-75 months). The mean age at the time of placement was 48 years (range 16-77 years). Altogether, 13 patients were male $(43 \%)$ and 12 patients $(40 \%)$ were African American. A group of $28(93 \%)$ required femoral $A V$ grafts owing to lack of upper extremity vascular outflow, 1 patient because of lack of upper extremity inflow, and 1 because of inability to control his arm during dialysis.

As expected, the patients had significant medical comorbidities (Table 1). The most common cause of renal failure was diabetes (8 patients, $27 \%$ of total). Other common etiologies of renal failure included hypertension (3 patients, 10\%), congenital nephropathy (3 patients, $10 \%$ ), obstructive uropathy (3 patients, $10 \%$ ), chronic pyelonephritis (2 patients, $7 \%$ ), medication toxicity ( 2 patients, $7 \%$ ), nephrolithiasis (2 patients, $7 \%$ ), and hemolytic-uremic syndrome (2 patients, $7 \%$ ).

The median number for previous access operations was 3 [interquartile range (IQR) 1-5]. The common femoral artery was used for inflow in two-thirds of the cases and the superficial femoral artery in one-third. The common femoral vein was used for outflow in 16 cases (55\%), the saphenofemoral junction in 9 cases $(31 \%)$, and the saphenous vein in 4 cases $(14 \%)$. The vessels chosen for the procedure had no significant effect on thrombosis, infection, or ischemia. Intravenous heparin was used prior to arterial occlusion. Generally, antiplatelet or anticoagulation therapy was resumed only if the patient had been on it preoperatively or if we documented a hypercoagulable state.

The perioperative (within 30 days of operation) complications are detailed in Table 2. The most common perioperative complication was graft thrombosis $(n=5$, $17 \%$ of patients).

The median time from graft placement to first graft failure was 81 days $(n=18$, IQR 42-196). The 1-year secondary graft patency rate was $41 \%$ (12 patients), the 2 -year rate was $26 \%$ (9 patients), and the 3 -year rate was $21 \%$ (6 patients). Actuarial secondary graft patency is detailed in Figure 1. The follow-up time is the interval to 
Table 2.

Perioperative complications

\begin{tabular}{lc}
\hline \multicolumn{1}{c}{ Complication } & No. \\
\hline None & $16(53 \%)$ \\
Thrombosis & $5(17 \%)$ \\
Admit for medical co-morbidities & $4(13 \%)$ \\
Infection & $2(7 \%)$ \\
Ischemia & $1(3 \%)$ \\
Cellulitis & $1(3 \%)$ \\
Complex arterial reconstruction & $1(3 \%)$ \\
\hline
\end{tabular}

final graft failure; or if there was no specific final graft failure, it was the interval to death (censored event).

Seven patients (23\%) underwent one operation/intervention to salvage graft function, four patients $(13 \%)$ underwent two salvage operations/interventions, and three patients $(10 \%)$ underwent three or more operative attempts at salvage. Among the patients who had salvage procedures $(n=14)$, the types of procedures done are shown in Table 3.

Among the patients with graft failure (primary graft failure, $n=18$; secondary graft failure, $n=16$ ), the sources of primary and secondary failure, respectively, were infection (6 patients, 33\%; 8 patients, 50\%), thrombosis (10 patients, 56\%; 7 patients, $44 \%$ ), and ligation (2 patients, $11 \%$; 1 patient, $6 \%$ ). Infection was the cause of final graft loss for $27 \%$ of the total grafts placed.

Patient survival was quite low: 1 year $81 \%, 2$ years $68 \%$, and 3 years $54 \%$. Actuarial patient survival is shown in Figure 2. At the conclusion of the study, nine patients $(27 \%)$ were alive with a failed graft, and seven patients $(23 \%)$ were alive with a functioning graft. Among those who died $(n=14)$, the mean time from femoral graft placement to death was $31.2 \pm 27.5$ months.

\section{DISCUSSION}

A modest number of studies on femoral $\mathrm{AV}$ grafts for hemodialysis access have reported a relatively good outcome for this type of access. ${ }^{5-7}$ One study reported a 1-year secondary patency rate of $83 \%$ with an infection rate of $22 \%{ }^{1}$ Another study reported a 1-year secondary patency rate of $73 \%$, with only 2 of 37 grafts becoming infected. $^{2}$ This study concluded that femoral AV grafts may be preferred to upper extremity AV grafts.

In contrast, there are reports of relatively poor outcomes with femoral AV grafts. The largest series on femoral AV grafts for hemodialysis $(n=134)$ reported a 1 -year graft survival rate of $62 \%$ (83 patients) with a $46 \%$
Table 3.

Graft salvage procedures

\begin{tabular}{ll}
\multicolumn{1}{c}{ Procedure } & No. \\
\hline Graft thrombectomy & $6(43 \%)$ \\
Revision & $5(36 \%)$ \\
Lymphocele drainage & $3(21 \%)$ \\
Reexploration & $2(14 \%)$ \\
Hematoma evacuation & $1(7 \%)$ \\
Above-knee amputation & $1(7 \%)$ \\
Lower extremity thrombectomy & $1(7 \%)$ \\
Incision and drainage of an abscess & $1(7 \%)$ \\
\hline
\end{tabular}

infection rate. ${ }^{3}$ Another series reported a primary patency rate of only $34 \%$ and a secondary patency rate of $68 \%$ at 1 year. This series also reported significant infection in $41 \%$ of the grafts. ${ }^{4}$ The authors concluded that femoral access can provide durable access in selected patients with few other access options but suggested that a tunneled, cuffed catheter should be considered in high risk patients (morbidly obese patients and those with limited survival).

It is difficult to compare these retrospective series, as methods of reporting differ significantly. The patients in our report had worse outcomes than did those in other series in the literature. Our secondary patency rate was only $41 \%$ at 1 year, with more than half of the patients requiring reoperation for graft salvage. These results are likely attributable to patient selection and referral patterns to our tertiary center. Publication bias may also have contributed to our results comparing poorly to those in the literature. Also, patients who undergo femoral access surgery at our center have exhausted all other access options, are usually long-term dialysis patients, and suffer from many severe medical co-morbidities (Table 1). Almost half of the patients had a significant perioperative surgical complication, with graft thrombosis (5 patients, 17\%) being the most common (Table 2). These patients frequently were morbidly obese, which has been previously reported to be a risk factor for early access failure. ${ }^{4}$ It is also interesting that four of our patients (13\%) required postoperative admission to address an urgent medical issue. The fact that general anesthesia was used in most of the cases likely contributed to some of these admissions. Infection (grade 2 infection, SVS/AAVS reporting standards) was also a significant problem in our patients, with eight grafts $(27 \%)$ lost to infection. These infections were rarely perioperative $(n=2 ; 7 \%$ of all cases) and presumably were the result of percutaneous access. Obesity was not noted to be a risk factor for infection, but definitive conclusions are difficult because of the small size of the series. We observed, as have other groups, that femoral access is more frequent in 


\section{Graft patency}

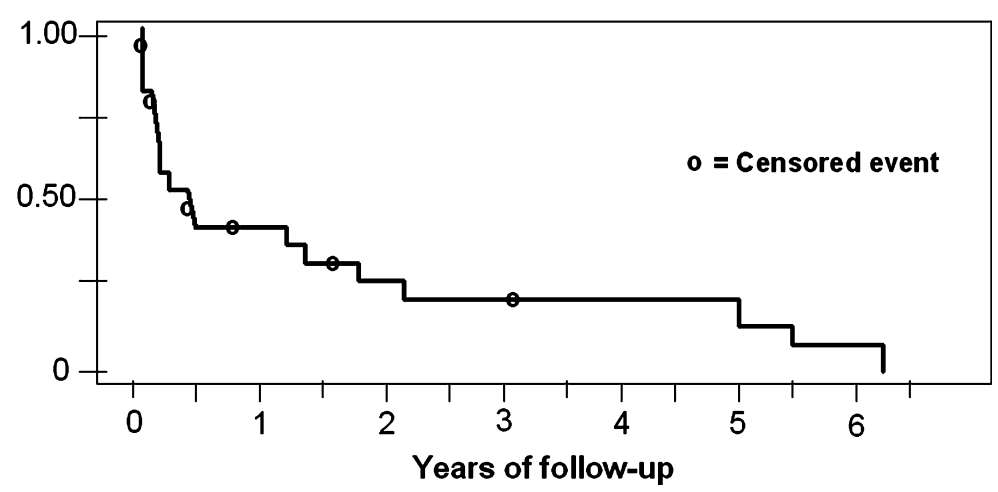

Survival

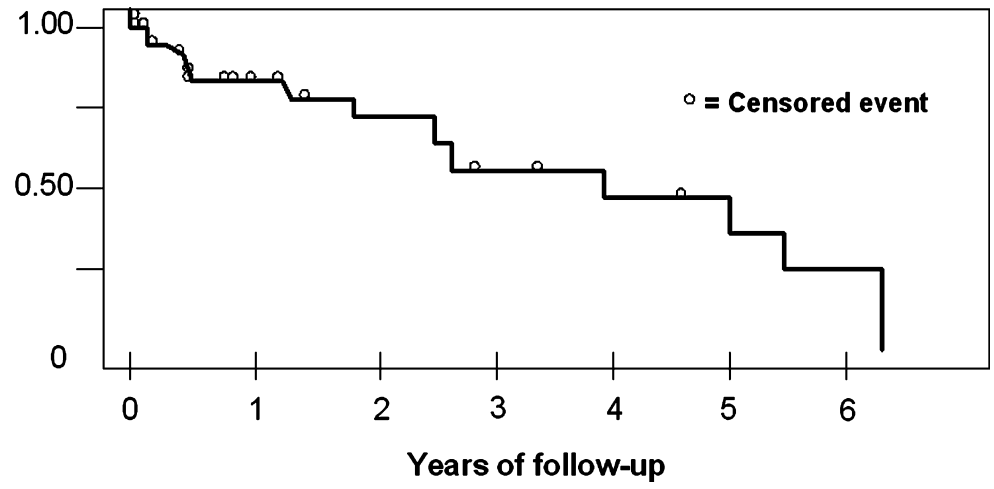

Figure 1. Secondary graft patency.

Figure 2. Patient survival.

women, presumably because women are smaller and have more problems with upper extremity venous outflow. ${ }^{2-4}$

The mean time from graft placement to death was less than 3 years. Although no deaths were directly attributable to complications of access surgery, this is obviously a high risk group. Similar mortality rates have been reported in other series. ${ }^{4,5}$

The National Kidney Foundation Guidelines do not favor catheters for hemodialysis access, stating that fewer than $10 \%$ of chronic hemodialysis patients should be maintained via catheters. ${ }^{8}$ The reason for such disfavor is the poor blood flow through the catheter with resultant inadequate hemodialysis and an increased rate of systemic infection with the need for hospitalization compared to AV grafts. ${ }^{9-12}$ These guidelines are based on comparisons between catheters and upper extremity AV grafts and as a result do not apply to the patients who present for femoral AV graft placement. For tunneled, cuffed catheters, the recommended target rate of systemic infection is less than $10 \%$ at 3 months and less than $50 \%$ at 1 year. Catheter malfunction is common but easily managed. ${ }^{8}$
In our experience, it is uncommon that interventional radiologists are unable to place a cuffed, tunneled internal jugular or subclavian vein hemodialysis catheter, even in the most complex patients. If the only option is a femoral AV graft or a cuffed, tunneled femoral catheter, the AV graft is generally a better option owing to high rates of infection in chronic indwelling femoral catheters. ${ }^{13}$

In many high risk patients (estimated short life expectancy, morbid obesity, severe peripheral vascular occlusive disease), an upper extremity indwelling catheter may be a better option when the overall operative risks and outcomes of femoral AV grafts are considered. Unfortunately, we do not have detailed data on the outcomes of a well matched cohort of patients who underwent hemodialysis via a cuffed catheter.

\section{CONCLUSIONS}

Patients who have exhausted their upper extremity hemodialysis options do poorly and have high complication rates. Consideration should be given to catheter- 
based access in certain high risk patients. A randomized multiinstitutional trial is needed to determine specific groups of patients who would benefit from a femoral graft rather than a cuffed catheter. We hope that similar single center reviews can provide the initiative and background information necessary for such future investigations.

\section{REFERENCES}

1. Tashjian DB, Lipkowitz GS, Madden RL, et al. Safety and efficacy of femoral-based hemodialysis access grafts. J Vasc Surg 2002;35:691-693.

2. Korzets A, Ori Y, Baytner S, et al. The femoral artery-femoral vein polytetrafluoroethylene graft: a 14-year retrospective study. Nephrol Dial Transplant 1998;13:1215-1220.

3. Vogel KM, Martino MA, O'Brien SP, et al. Complications of lower extremity arteriovenous grafts in patients with endstage renal disease. South Med J 2000;93:593-595.

4. Cull JD, Cull DL, Taylor SM, et al. Prosthetic thigh arteriovenous access: outcome with SVS/AAVS reporting standards. J Vasc Surg 2004;39:381-386.

5. Khadra MH, Dwyer AJ, Thompson JF. Advantages of polytetrafluoroethylene arteriovenous loops in the thigh for hemodialysis access. Am J Surg 1997;173:280-283.
6. Bhandari S, Wilkinson A, Sellars L. Saphenous vein forearm grafts and GoreTex thigh grafts as alternative forms of vascular access. Clin Nephrol 1995;44:325-328.

7. Taylor SM, Eaves GL, Weatherford DA, et al. Results and complications of arteriovenous access dialysis grafts in the lower extremity: a five year review. Am Surg 1996;62:188191.

8. National Kidney Foundation/DOQI Clinical Practice Guidelines for Vascular Access: Update 2000 (www.Kidney.org).

9. Moss $A H$, McLaughlin MM, Lempert KD, et al. Use of a silicone catheter with a Dacron cuff for dialysis short-term vascular access. Am J Kidney Dis 1988;12:492-498.

10. Moss $A H$, Vasilakis $C$, Holley $\mathrm{JL}$, et al. Use of a silicone dual-lumen catheter with a Dacron cuff as a long-term vascular access for hemodialysis patients. Am J Kidney Dis 1990;16:211-215.

11. Ena J, Boelaert JR, Boyken LD, et al. Epidemiology of Staphylococcus aureus infections in patients on hemodialysis. Infect Control Hosp Epidemiol 1994;15:78-81.

12. Hoen B, Kessler M, Hestin D, et al. Risk factors for bacterial infections in chronic haemodialysis adult patients: a multicentre prospective survey. Nephrol Dial Transplant 1995;10:377-381.

13. Oliver MJ, Callery SM, Thorpe KE, et al. Risk of bacteremia from temporary hemodialysis catheters by site of insertion and duration of use: a prospective study. Kidney Int 2000;58:2543-2545. 